\title{
Sessile serrated adenoma/polyps with a depressed surface: a rare form of sessile serrated adenoma/polyp
}

\author{
Eun-Jung Lee ${ }^{1}$, Mi-Jung Kim² ${ }^{*}$, Sung-Min Chun ${ }^{3}$, Se-Jin Jang ${ }^{3}$, Do Sun Kim ${ }^{1}$, Doo Han Lee ${ }^{1}$ and Eui Gon Youk
}

\begin{abstract}
Sessile serrated adenoma/polyps (SSA/Ps) usually appear flat to sessile with a smooth-appearing surface. However, macroscopic appearances of SSA/P can vary from flat-elevated to nodular and can even show a pedunculated configuration as we previously reported. The aim of the current study was to evaluate the clinicopathologic features of another under-recognized form of SSA/P which shows a depressed surface. Among 634 cases of sessile serrated adenoma/polyp, a total of seven sessile serrated adenoma/polyps showing a depressed surface were identified in 6 patients during the review of endoscopic images between January 2013 and November 2013. One of these was found during the review of previous endoscopic images of the same patient. Patients were more often middle-aged to elderly men (83.3 \%) and had synchronous conventional adenomas and/or SSA/Ps except for one man. The polyps usually occurred in the proximal colon (71.4\%) and the mean size of polyps was $9.3 \mathrm{~mm}$ (range; 6-13 mm). Most cases (71.4 \%) were of a flat-elevated type, and the remaining polyps (28.6\%) were sessile. The majority of polyps (85.7 \%) showed a mucus cap. All but one of the cases (85.7 \%) showed BRAF-V600E mutations. Our findings are that SSA/Ps can show a central depression although such cases are rare. The endoscopic and clinicopathologic features of SSA/PS showing a depressed surface appear to be similar to usual SSA/Ps except for the presence of a depressed surface and marked male preponderance.
\end{abstract}

Virtual slides: The virtual slide(s) for this article can be found here: http://www.diagnosticpathology.diagnomx.eu/vs/ 1562070886167874.

Keywords: Sessile serrated adenoma, Colon, Endoscopy, Serrated polyp

\section{Background}

With the understanding of the biologic nature of sessile serrated adenoma/polyps (SSA/Ps), its endoscopic detection has become more important $[1,2]$. SSA/Ps are usually sessile to flat and similar in color to the surrounding mucosa. The presence of a mucus cap, a rim of bubbles or debris, alteration of the contour of a mucosal fold, and/or loss of the normal mucosal vascular pattern also suggests a possibility of an SSA/P [3]. Recently, the type II-O pit pattern has been introduced as a hallmark of an SSA/P [4]. However, some kinds of SSA/Ps are still under-recognized and poorly characterized despite increasing recognition of SSA/Ps among both endoscopists

\footnotetext{
* Correspondence: mjkimdoc@gmail.com

${ }^{2}$ Department of Pathology, Daehang Hospital, 481-10 BangBae3-dong,

Seocho-gu 137-820, Seoul, Republic of Korea

Full list of author information is available at the end of the article
}

and pathologists. In a previous study, we described a pedunculated serrated polyp with histologic features of an SSA/P that could represent a pedunculated form of $\mathrm{SSA} / \mathrm{P}$ in the spectrum of serrated neoplasia [5].

Herein we present seven cases of a previously undescribed form of SSA/P that shows a depressed surface. To the best of our knowledge, SSA/P with a depressed surface has not been reported in the English literature. We are reporting this rare form of SSA/Ps with the results of clinicopathologic and molecular analysis.

\section{Case presentation}

Table 1 lists the demographic and clinical information for the cases. All but one case were obtained from men. The mean age of seven cases was 57.3 years (range, 40-66). Most (71.4 \%) polyps were removed from the proximal colon except for two cases from the sigmoid- 
Table 1 Clinicopathologic and molecular features of SSA/Ps showing a depressed surface

\begin{tabular}{|c|c|c|c|c|c|c|c|c|}
\hline Case & Age (years) & Sex & Location & Size (mm) & Endoscopic findings $^{\mathrm{a}}$ & Procedure & Previous biopsy & BRAF mutation \\
\hline 1 & 40 & M & Ascending colon & 10 & 0-\|la + Ilc, covered with mucus & EMR & - & V600E \\
\hline 2 & 59 & M & Transverse colon & 10 & 0-\|la + Ilc, covered with mucus & Polypectomy & - & V600E \\
\hline 3 & 50 & M & SD junction & 8 & $0-|s+| \mid c$ & EMR & + & V600E \\
\hline 4 & 65 & M & SD junction & 13 & 0 -Is + IIc, covered with mucus & EMR & + & V600E \\
\hline 5 & 66 & $\mathrm{~F}$ & Ascending colon & 11 & 0-\|la + Ilc, covered with mucus & EMR & + & V600E \\
\hline 6 & 62 & M & Transverse colon & 6 & 0-\|la + Ilc, covered with mucus & Polypectomy & - & Negative \\
\hline 7 & 59 & M & Splenic flexure & 7 & $0-\|a+\| c$, covered with mucus & EMR & - & V600E \\
\hline
\end{tabular}

F, female; $M$, male; SD, sigmoid-descending; EMR, endoscopic mucosal resection

${ }^{\text {a }}$ Paris classification [7]

descending junction. The polyps were relatively small, with a mean size of $9.3 \mathrm{~mm}$ (range, 6-13). Five of them were accompanied by synchronous tubular adenomas and two cases were associated with synchronous SSA/Ps. BRAF mutations were observed in all but one case of SSA/P showing a central depression. All mutations were a single missense mutation in codon 600 of exon 15 (V600E) (Fig. 1).

\section{Materials and methods}

A total of seven SSA/Ps showing a depressed surface were identified in six patients among 634 cases of SSA/P during the review of endoscopic images between January 2013 and November 2013. Among them, one case (case 7) was found during the review of previous endoscopic images of case 6. Patients were assessed for clinicopathologic features, including age and gender, anatomic site and size of polyp, and the presence and type of synchronous or metachronous polyps upon follow-up by reviewing the patients' medical records, endoscopy and pathology reports. Permission for the study was obtained from the institutional review board at Daehang Hospital.

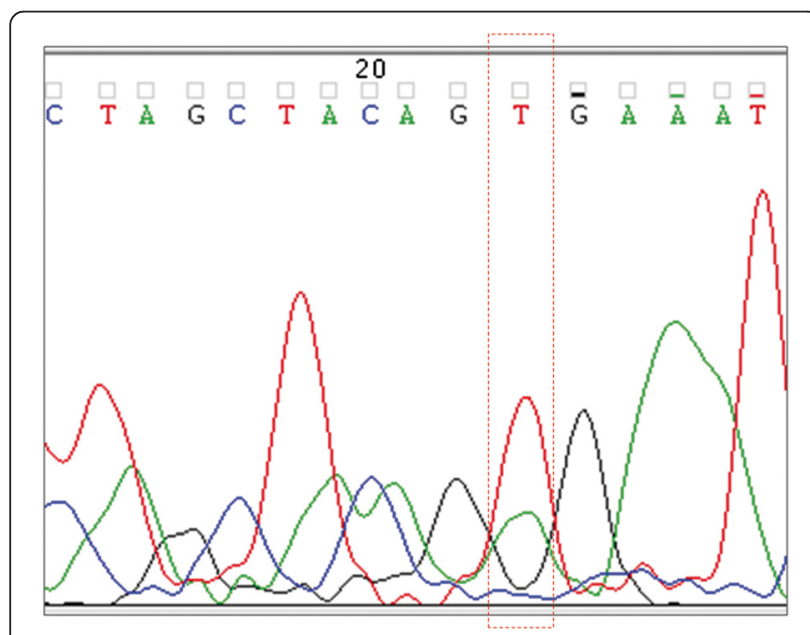

Fig. 1 Representative example of sequencing result for BRAF. There was a GTG-to-GAG change (V600E) at codon 600 of exon 15

\section{Histology and mutation study}

All specimens were routinely processed, stained with hematoxylin and eosin, and evaluated. The polyps included in this study were reviewed by M.J.K., who specializes in gastrointestinal pathology. SSA/Ps were defined by the abnormal architecture of the crypt bases, a so called "abnormal proliferation", such as the branching of crypts, dilation of the base of the crypts, and inverted T- or L-shaped crypts [1, 2].

Genomic DNA was extracted from formalin-fixed, paraffin-embedded specimens. After DNA extraction, PCR amplification was performed as previously described $[5,6]$. The amplified DNA products were purified and then direct sequencing was carried out using an ABI PRISM 310 sequence analyzer (Applied Biosystems, Foster City, CA).

\section{Colonoscopy}

Colonoscopy was performed after standard bowel preparation. Total colonoscopies were carried out using a singlechannel HD colonoscope (Olympus CF-H260AI; Olympus Optical, Tokyo, Japan). When a lesion was detected by colonoscopic examination, the surface mucus was washed away, and indigo carmine dye was spread over the lesion. The endoscopist (E.J.L.) retrospectively reviewed endoscopy images of the histologically proven SSA/Ps diagnosed between January 2013 and November 2013, reevaluating the morphology of polyps for size, anatomic location, gross appearance, and the presence of a mucous cap. The macroscopic morphology of polyps was characterized according to the Paris endoscopic classification (protruding sessile (Is) and protruding pedunculated (Ip); non-protruding superficial elevated (IIa), flat (IIb), and depressed (IIc) [7]. In this study, all the non-protruding neoplasms (IIa, IIb, IIc) were categorized as "flat". The anatomic locations of polyps were grouped as proximal colon (cecum to splenic flexure) vs. distal colon and rectum combined.

\section{Results}

The clinicopathologic and molecular features of the patients with SSA/Ps showing a depressed surface are 
summarized in Table 1. Patients were more often middle-aged to elderly men (83.3\%). Most (71.4 \%) polyps were removed from the proximal colon except for two cases from the sigmoid-descending junction. The polyps tended to be relatively small, with a mean size of $9.3 \mathrm{~mm}$ (range; $6-13 \mathrm{~mm}$ ). Five of them were accompanied by synchronous tubular adenomas and two cases were associated with synchronous SSA/Ps. BRAF mutations were observed in all but one case. All mutations were a single missense mutation in codon 600 of exon 15 (V600E) (Fig. 1).

All polyps were slightly elevated to sessile. The surface of the polyps was relatively smooth with a depressed surface (Fig. 2a,b,c). Five polyps showed a single depressed focus in the central portion, while the remaining two cases displayed surface depression in more than one area. The color of the polyps was slightly pale or the same as the normal mucosa. All but one of the polyps were covered with a mucus cap. The polyps showed an irregular or vague margin. Five $(71.4 \%)$ cases were removed by endoscopic mucosal resection and two were obtained by polypectomy.

On histopathological examination, both the flat-elevated and depressed areas showed dilatation and branching of the basal crypts, satisfying the criteria of an SSA/P (Fig. 2d). Histologic features suggesting other polyp or dysplasia were not noted in any of the cases. Histologic evidence of cytologic dysplasia such as nuclear hyperchromasia and elongation, pseudostratification, apoptosis, increased mitotic activity, and loss of mucin was not noted in any case.

\section{Discussion}

In the current study, we describe 'SSA/Ps of the colon with a depressed surface' which share most clinicopathologic features with usual SSA/Ps. Most of these polyps are right-sided, covered with a mucous cap, and show BRAF-V600E mutations. In contrast to the tendency of usual SSAPs in women, these polyps frequently occurred in men [8].

According to the Paris classification [7], SSA/Ps with a depressed surface correspond to a type 0-IIa + IIc or 0 -Is + IIc. While depressed type colorectal lesions have been reported to have greater risk of developing highgrade dysplasia or submucosal invasive cancer than other types [7, 9], there was no evidence of dysplasia in any case of SSA/P with a depressed surface in this study. However, larger-scale studies would be needed to confirm the prevalence rate of dysplasia and biologic nature of these polyps.

Besides under-recognition, there is also a potential risk for misdiagnosis as these polyps can resemble inverted colonic diverticulum (ICD), and vice versa [10]. Before spraying indigo carmine dye on the mucosal surface, the surface of these polyps can show only minimal mucosal changes. Therefore, if there is no knowledge about this kind of polyp, endoscopists can mistake this lesion for
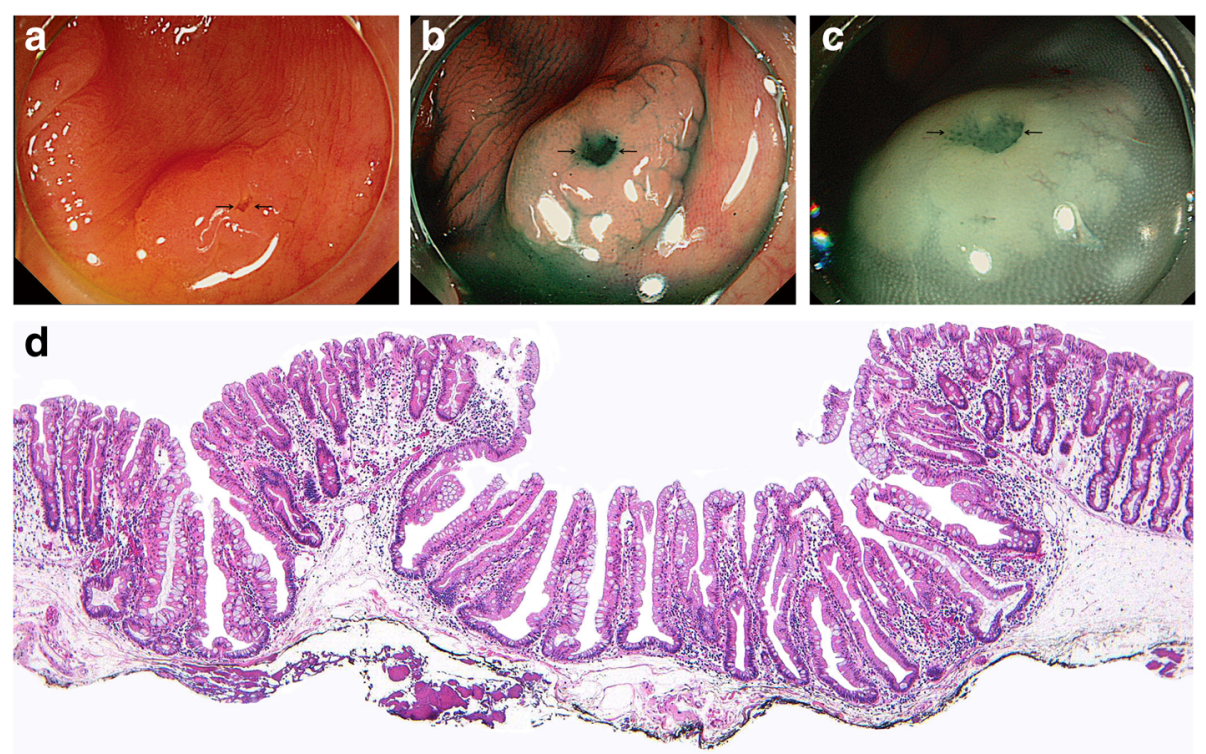

Fig. 2 Endoscopic and histologic findings of case 1. a: Colonoscopy displayed a $10 \mathrm{~mm}$ laterally spreading tumor in the ascending colon. Most of the area of the polyp showed a flat-elevated surface with a mucous covering while the central portion displayed a depressed appearance (black arrows). $\mathbf{b}$ and $\mathbf{c}$ : Endoscopic images after the spraying of indigo carmine dye revealed a type II pit pattern in both the elevated and depressed areas (black arrows). d: A microscopic image showed typical histologic features of an SSA/P, such as dilatation and branching of basal crypts except for a central depression (H\&E staining, x40) 
an ICD because these polyps can appear as a shiny, smooth-surfaced, polypoid lesion with a central indentation [10]. Although both lesions are rare, endoscopists should keep in mind both of these entities, as different approaches should be used depending on the judgement during endoscopy. If one favors an SSA/P, a complete removal of the lesion should be considered. However, in the case of ICD, an inadvertent attempt to remove the ICD endoscopically can cause serious complications, such as perforation [10].

In the current study, three of seven cases underwent a previous biopsy before EMR or polypectomy. Compared to the original shape, the central depression of the polyps was less noticeable in post-biopsy endoscopic images and polypectomy specimens. We suppose that a post-biopsy healing process results in biopsy scar and resultingly an architectural change in polyps. Therefore, previous endoscopic images need to be reviewed not to miss an SSA/P with a depressed surface if the patient had undergone a previous biopsy.

Finally, we suppose that the terminology "SSA/P" still has some drawbacks because the macroscopic appearance of SSA/Ps is not always sessile. Therefore, a new terminology reflecting their genuine nature might be necessary to reduce confusion in a clinical setting.

\section{Conclusion}

We report the clinicopathologic and molecular features of a previously undescribed form of SSA/P. This type of polyp represents less than $1 \%$ of all serrated polyps in our study. Clinically, SSA/Ps showing a depressed surface tend to develop in middle-aged to elderly patients, predilect for men, often occur in patients with conventional adenomas, usually arise in the proximal colon, and show BRAF-V600E mutations. The clinicopathologic features of SSA/Ps with a depressed surface seem to be similar to usual SSA/Ps except for the marked male preponderance and a depressed surface. However, a large-scale study would be necessary to confirm our observations because there is little data on this point.

\section{Consent}

Written informed consents were obtained from the reviewed patients for publication of this case report and accompanying images. A copy of written consent is available for review by the Editor-in Chief of this Journal.

\section{Abbreviations}

EMR: Endoscopic mucosal resection; ICD: Inverted colonic diverticulum; SSA/P: Sessile serrated adenoma/polyp.

\section{Competing interests}

The authors declare that they have no competing interests.

\section{Authors' contributions}

MJK is the principal researcher and performed the histopathological examination. EJL drafted the manuscript. DSK, DHL, and EGU were responsible for the clinical data. SMC and SJJ carried out the mutation analysis. All authors read and approved the final version submitted.

\section{Author details}

${ }^{1}$ Department of Surgery, Daehang Hospital, Seoul, Republic of Korea. ${ }^{2}$ Department of Pathology, Daehang Hospital, 481-10 BangBae3-dong, Seocho-gu 137-820, Seoul, Republic of Korea. ${ }^{3}$ Department of Pathology, University of Ulsan College of Medicine, Asan Medical Center, Poongnap

2-Dong, Songpa-Gu, Seoul 138-736, Republic of Korea.

Received: 13 April 2015 Accepted: 12 June 2015

Published online: 20 June 2015

\section{References}

1. Bosman FT, Carneiro F, Hruban RH, Theise ND. WHO Classification of Tumours of the Digestive System. 4th ed. Lyon, France: IARC Press; 2010. p. 160-5.

2. Torlakovic E, Skovlund E, Snover DC, Torlakovic G, Nesland JM. Morphologic reappraisal of serrated colorectal polyps. Am J Surg Pathol. 2003;27:65-81.

3. Tadepalli US, Feihel D, Miller KM, Itzkowitz SH, Freedman JS, Kornacki S, et al. A morphologic analysis of sessile serrated polyps observed during routine colonoscopy (with video). Gastrointest Endosc. 2011;74:1360-8.

4. Kimura T, Yamamoto E, Yamano HO, Suzuki H, Kamimae S, Nojima M, et al. A novel pit pattern identifies the precursor of colorectal cancer derived from sessile serrated adenoma. Am J Gastroenterol. 2012;107:460-9.

5. Kim MJ, Lee EJ, Chun SM, Jang SJ, Kim CH, Seo JP, et al. Pedunculated serrated polyp with histologic features of sessile serrated adenoma: a clinicopathologic and molecular study. Am J Surg Pathol. 2013;37:1039-43.

6. Kim MJ, Lee EJ, Suh JP, Chun SM, Jang SJ, Kim do S, et al. Traditional serrated adenoma of the colorectum: clinicopathologic implications and endoscopic findings of the precursor lesions. Am J Clin Pathol. 2013;140:898-911.

7. The Paris endoscopic classification of superficial neoplastic lesions: esophagus, stomach, and colon: November 30 to December 1, 2002 Gastrointest Endosc. 2003:58:S3-43. Review.

8. Spring KJ, Zhao ZZ, Karamatic R, Walsh MD, Whitehall VL, Pike T, et al. High prevalence of sessile serrated adenomas with BRAF mutations: a prospective study of patients undergoing colonoscopy. Gastroenterology. 2006;131:1400-7.

9. Matsuda T, Saito Y, Hotta K, Sano Y, Fujii T. Prevalence and clinicopathological features of nonpolypoid colorectal neoplasms: should we pay more attention to identifying flat and depressed lesions? Dig Endosc. 2010;22 Suppl 1:S57-62.

10. Yusuf SI, Grant C. Inverted colonic diverticulum: a rare finding in a common condition? Gastrointest Endosc. 2000;52:111-5.

\section{Submit your next manuscript to BioMed Central and take full advantage of:}

- Convenient online submission

- Thorough peer review

- No space constraints or color figure charges

- Immediate publication on acceptance

- Inclusion in PubMed, CAS, Scopus and Google Scholar

- Research which is freely available for redistribution 